\title{
SOME THEOREMS ON THE EULER $\phi$-FUNCTION
}

\author{
N. G, GUNDERSON
}

The Euler $\phi$-function, $\phi(m)$, denotes the number of positive integers not greater than $m$ which are relatively prime to $m .^{1}$ It was noted by U. Scarpis ${ }^{2}$ that $n \mid \phi\left(p^{n}-1\right)$. Generalizations of this result are obtained in Theorems 9 and 10 .

The first five theorems are either well known or self-evident. ${ }^{3}$

Theorem 1. If $p_{1}, \cdots, p_{k}$ are the distinct prime factors of $m$, then

$$
\phi(m)=m\left(p_{1}-1\right)\left(p_{2}-1\right) \cdots\left(p_{k}-1\right) / p_{1} p_{2} \cdots p_{k} .
$$

THEOREM 2. If $a_{1}, \cdots, a_{k}$ are relatively prime in pairs, then

$$
\phi\left(a_{1} \cdots a_{k}\right)=\phi\left(a_{1}\right) \cdot \phi\left(a_{2}\right) \cdots \phi\left(a_{k}\right) .
$$

THEOREM 3. If $w$ is the product of the distinct prime factors common to $m$ and $n$, then

$$
\phi(m n)=w \cdot \phi(m) \cdot \phi(n) / \phi(w) .
$$

Theorem 4. If $a \mid b$, then $\phi(a) \mid \phi(b)$.

Theorem 5. If $q \mid a$ and $q \equiv 1\left(\bmod p^{\alpha}\right)$, then $p^{\alpha} \mid \phi(a)$.

Theorem 6. If $p$ is an odd prime, $a \neq b$, and $\alpha \geqq 1$, then

$$
p^{2 \alpha-1} \mid \phi\left(a^{p^{\alpha}}+b^{p^{\alpha}}\right) \text {. }
$$

The proof is by induction on $\alpha$. We assume $a>b$. In the notation of Birkhoff and Vandiver, ${ }^{4} a^{p}+b^{p}=V_{2 p} / V_{p}$. By their Theorems $\mathrm{V}$ and $\mathrm{I}$, there is a prime divisor $q$ of $a^{p}+b^{p}$ such that $q \equiv 1(\bmod p)$ unless $p=3, a=2, b=1$. Then by Theorem $5, p \mid \phi\left(a^{p}+b^{p}\right)$, and in the exceptional case, $3 \mid \phi\left(2^{3}+1^{3}\right)$. Thus the theorem holds for $\alpha=1$, starting the induction, so we assume it for all positive integers less than $\alpha$. We adopt the notation $C=A B$, where

$$
\begin{gathered}
C=a^{p^{\alpha}}+b^{p^{\alpha}}, \quad P=p^{\alpha-1}, \quad A=a^{p}+b^{p}, \\
B=a^{(p-1) P}-a^{(p-2) P} \cdot b^{P}+\cdots-a^{P} \cdot b^{(p-2) P}+b^{(p-1) P} .
\end{gathered}
$$

Received by the editors June 12, 1942.

${ }^{1}$ In this discussion all letters represent positive integers. In particular, $p$ and $q$ represent primes.

2 Period. Mat. vol. 29 (1913) p. 138.

${ }^{3}$ See, for example, L. E. Dickson, History of the theory of numbers, vol. 1, chap. 5.

4 Ann. of Math. (2) vol. 5 (1903) pp. 173-180. 
Case 1. $(a, b)=1$.

Again using the notation of Birkhoff and Vandiver, $B=C / A$ $=\left(V_{2 p P} / V_{p P}\right)\left(V_{P} / V_{2 P}\right)$, so we see by their Theorems $\mathrm{V}$ and $\mathrm{I}$ that there is a prime divisor $q$ of $B$ such that $q \equiv 1\left(\bmod p^{\alpha}\right)$. Hence by Theorem 5 we have that $p^{\alpha} \mid \phi(B)$. By the hypothesis of induction we have $p^{2(\alpha-1)-1} \mid \phi(A)$.

Now, if $(A, B) \neq 1$, let $r$ be a common prime factor of $A$ and $B$. Then $a^{P} \equiv-b^{P}(\bmod r)$, so that $B \equiv p \cdot a^{(p-1) P} \equiv 0(\bmod r)$. If $r \mid a$, then $r \mid b$, contrary to $(a, b)=1$, so $r=p$. Then by Theorem 3 ,

$$
\phi(C)=p \cdot \phi(A) \cdot \phi(B) /(p-1),
$$

so we have that $p \cdot p^{2(\alpha-1)-1} \cdot p^{\alpha} \mid \phi(C)$. But since $\alpha>1,3 \alpha-2>2 \alpha-1$, so $p^{2 \alpha-1} \mid \phi(C)$.

If $(A, B)=1$, then $\phi(C)=\phi(A) \cdot \phi(B)$, and $p^{2(\alpha-1)-1} \cdot p^{\alpha} \mid \phi(C)$. Again, since $\alpha>1,3 \alpha-3 \geqq 2 \alpha-1$, and $p^{2 \alpha-1} \mid \phi(C)$.

Case 2. $(a, b) \neq 1$.

Let $(a, b)=c, a=c a_{1}, b=c b_{1},\left(a_{1}, b_{1}\right)=1$. Further, since $a>b, a_{1}$ and $b_{1}$ are not both 1 , and so $a_{1}>b_{1}$. By Case $1, p^{2 \alpha-1} \mid \phi\left(a_{1}^{p \alpha}+b_{1}^{p \alpha}\right)$ so by Theorem 4, $p^{2 \alpha-1} \mid \phi\left(a^{p^{\alpha}}+b^{p^{\alpha}}\right)$.

Theorem 7. If $a \neq b$, then $2^{\alpha+1} \mid \phi\left(a^{2 \alpha}+b^{2 \alpha}\right)$.

We note that $a^{2 \alpha}+b^{2 \alpha}=2^{\beta}$ would imply $a=b=2$, so $a^{2^{\alpha}}+b^{2^{\alpha}}$ has an odd factor, say $q$. For $(a, b)=1$, Euler ${ }^{5}$ has shown that $q \equiv 1\left(\bmod 2^{\alpha+1}\right)$, so by Theorem $5,2^{\alpha+1} \mid \phi\left(a^{2^{\alpha}}+b^{2^{\alpha}}\right)$. For $(a, b) \neq 1$ we proceed as in Case 2 of Theorem 6.

Theorem 8. If $a>b$, then $p^{2 \alpha-1} \mid \phi\left(a^{p^{\alpha}}-b^{p^{\alpha}}\right)$.

The proof of this theorem parallels that of Theorem 6 .

THEOREM 9. If $a>b$, and $m$ is the product of the distinct prime factors of $n$, then $\left(n^{2} / m\right) \mid \phi\left(a^{n}-b^{n}\right)$.

Let $n=P_{1} P_{2} \cdots P_{k}$ where $P_{i}=p_{i}^{\alpha_{i}}$. Then $a^{n}-b^{n}=\left(a^{n_{i}}\right)^{P_{i}}-\left(b^{n_{i}}\right)^{P_{i}}$ where $n_{i}=P_{1} \cdots P_{i-1} P_{i+1} \cdots P_{k}$. Then by Theorem $8, p_{i}^{2 \alpha_{i}-1} \mid \phi\left(a^{n}-b^{n}\right)$, and the theorem follows immediately.

Theorem 10. If $a \neq b$, then $n \mid \phi\left(a^{n}+b^{n}\right)$.

This proof parallels that of Theorem 9 .

Theorems 9 and 10 can be combined in various ways, for example, we have this theorem:

${ }^{5}$ Commentationes Arithmeticae Collectae, vol. 1, p. 55. See also Amer. Math. Monthly vol. 10 (1903) p. 171, misprint of $2 m$ for $2^{m}$. 
THEOREM 11. If $(a, b)=1$, and $m$ is the product of the distinct prime factors of $n$, then

$$
\left(n^{3} / m\right) \mid \phi\left(a^{2 n}-b^{2 n}\right) .
$$

CoRnell University

\title{
APPLICATIONS OF TRANSITIVITIES OF BETWEENNESS IN LATTICE THEORY ${ }^{1}$
}

\author{
M. F. SMILEY AND W. R. TRANSUE
}

Introduction. This paper solves three characterization problems for lattices $^{2}[1]$. Problem I is to characterize those metric spaces [2] into which lattice operations which are consistent with the given metric $[1$, p. 41] may be introduced. Problem II is to characterize those members of a rather general class of abstract systems which are modular lattices, while Problem III consists in the characterization of lattices in an even larger class of abstract systems:

Problem I has already been solved by V. Glivenko [3]. He showed that the property: "Among those elements metrically between $[4$, p. $76 ; 2$ ] two elements $a$ and $b$, the element $a \cup b$ is farthest from $O$," and its dual characterize those metric spaces which are also metric lattices with the same metric and least element $O$. Our approach to Problem I is through the existence of certain metric singularities [2, p. 47] in every metric lattice. Our solution also involves certain five point transitivities [5, Part I] of metric betweenness. The abstract system involved in Problem II (Problem III) is a wide generalization of the concept of a metric space-so general, in fact, that it also includes the concept of a modular lattice (lattice). We find it not difficult to extend the ideas essential to our solution of Problem I to give analogous solutions of Problems ${ }^{3}$ II and III. Briefly, our results consist in characterizing the three important systems: metric

Presented to the Society, April 4, 1942; received by the editors June 2, 1942.

1 This paper is an expansion of our note Metric lattices as singular metric spaces which was presented to the Society on December 29,1941, and which was to appear in Bull. Amer. Math. Soc.

2 Numbers in square brackets refer to the list of references at the end of the paper.

${ }^{3}$ It would be interesting to characterize metric spaces among our general systems. This problem appears difficult to us, and we make no attempt to solve it. 